Recibido el 30 de abril de 2021. Aceptado el 26 de octubre de 2021. Publicado el 11 de noviembre de 2021.

${ }^{*}$ Autor para correspondencia: Rafael Alarcón. Correo electrónico: ralarcon@colef.mx

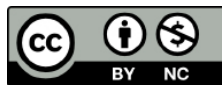

Esta obra está protegida bajo una Licencia Creative Commons Atribución-NoComercial 4.0 Internacional.

\section{Mexicanos deportados de Estados Unidos a Ciudad Juárez por conducir bajo la influencia del alcohol}

\section{Mexicans deported from the United States to Ciudad Juarez for driving under the influence of alcohol}

\author{
${ }^{a}$ Investigadora independiente, Tijuana, México, correo electrónico: \\ karenmuroarechiga@gmail.com \\ b El Colegio de la Frontera Norte, Departamento de Estudios Sociales, Tijuana, México, correo \\ electrónico: ralarcon@colef.mx
}

\section{Resumen}

En este artículo se analizan los factores legislativos, sociales y económicos que explican por qué un alto número de ciudadanos mexicanos han sido deportados formalmente de Estados Unidos bajo la acusación de conducir un vehículo bajo la influencia del alcohol. Para este fin, se analiza la transformación de la política de inmigración de Estados Unidos para examinar, a través de entrevistas semiestructuradas, los procesos de deportación de 23 hombres mexicanos que fueron expulsados a Ciudad Juárez, acusados de este delito en 2019. Los hallazgos revelan que los entrevistados tenían distintos niveles de consumo de alcohol y que la mayoría había iniciado dicha práctica en ese país. Se evidencia el papel protagónico de ofíciales de policías locales que actúan como "policías de inmigración" y el que a mayor tiempo de residencia de los inmigrantes en Estados Unidos mayor es la propensión a tener comportamientos de riesgo como el abuso del alcohol.

Palabras clave: migración, deportación, alcoholismo, México, Estados Unidos.

\section{Abstract}

This article analyzes the legislative, social, and economic factors that explain why a high number of Mexican citizens have been removed from the United States under the accusation of driving a vehicle under the influence of alcohol. To this end, the recent transformation of United States immigration policy is analyzed to examine, through semi-structured interviews, the deportation processes of 23 Mexican men who were expelled to Ciudad Juarez, accused of this crime in 2019. The findings reveal that the interviewees had different levels of alcohol consumption and that most of them had started this practice in the United

CÓMO CITAR: Muro Aréchiga, K. \& Alarcón, R. (2021). Mexicanos deportados de Estados Unidos a Ciudad Juárez por conducir bajo la influencia del alcohol [Mexicans deported from the United States to Ciudad Juarez for driving under the influence of alcohol]. Estudios Fronterizos, 22, e081. https://doi.org/10.21670/ref.2118081 
States. On the other hand, there is evidence of the leading role of local police officers acting as "immigration police" and that the longer immigrants reside in the United States, the greater their propensity to engage in risky behaviors such as alcohol abuse.

Keywords: migration, deportation, alcoholism, Mexico, United States.

\section{Introducción}

La deportación de ciudadanos mexicanos desde Estados Unidos de América (EUA) ha sido parte de la larga historia de la migración a ese país, sin embargo, este proceso ha adquirido características especiales desde el inicio del siglo xxI por su masividad, sus consecuencias legales a largo plazo, su composición social que incluye migrantes con una larga residencia en EUA y porque un alto porcentaje es deportado por la acusación de conducir un vehículo bajo la influencia del alcohol u otras drogas, delito que se denomina driving under the influence (DUI) en EUA.

De acuerdo con el análisis de los datos de TRAC Immigration (2021) de Syracuse University para los años fiscales de 2004 a 2020, conducir un vehículo motorizado bajo la influencia del alcohol es el primer delito de una larga lista de ofensas que condujeron a la deportación de una muestra de 1000462 migrantes mexicanos. De ellos, 335 174, uno de cada tres (33.5\%), fue deportado sin haber sido convicto de ningún delito. De los 665288 que fueron convictos de un crimen, 66 246, migrantes mexicanos deportados fueron convictos por conducir un vehículo motorizado bajo la influencia del alcohol u otras drogas que equivale a 9.9\%. Del resto, 225862 (22.6\%) fueron convictos de alguno de los siguientes nueve delitos: agresión, infracción de tráfico, drogas peligrosas, ingreso ilegal a una propiedad, venta de cocaína, posesión de cocaína, entrada ilegal a EUA, venta de marihuana y robo. Los 373180 restantes $(37.3 \%)$ fueron deportados por otros delitos.

Por su parte, Coubès (2018) utiliza estadísticas sobre repatriados mexicanos de Estados Unidos en la Encuesta sobre Migración de la Frontera Norte (Emif Norte) y encuentra que, en 2017, 29\% de los deportados mexicanos residentes en EuA fueron detenidos por manejar alcoholizados o bajo la influencia de otra droga. Daniel Rodríguez, abogado en inmigración en Arizona, afirma que las autoridades migratorias están incrementando los arrestos de inmigrantes por violaciones relacionadas con conducir en estado de ebriedad,

sin importar si fueron detenidos por una infracción menor o si fue en el pasado cuando ocurrió alguna detención por Dui. Para la corte, el individuo se vuelve un peligro para la comunidad y un caso criminal y las autoridades se enfocan más en el perfil racial para negar la fianza. (EFE, 2019)

Esta investigación tiene como objetivo principal analizar los factores legislativos, sociales y económicos que explican la razón por la que un alto número de ciudadanos mexicanos han sido deportados formalmente desde euA con la acusación de conducir un vehículo bajo la influencia del alcohol. Para este fin, se exploran las teorías que podrían apoyar el análisis de este proceso, así como la transformación de la reciente política de inmigración de EuA que ha facilitado la deportación formal de una gran cantidad de extranjeros que no tienen la ciudadanía estadounidense y que ha afectado principalmente a los ciudadanos mexicanos. Estos antecedentes se utilizan para 
analizar la investigación empírica en la que se examinan los procesos de deportación de 23 hombres mexicanos que fueron removidos de EuA a Ciudad Juárez acusados de Dui en 2019. La ciudad fronteriza de Ciudad Juárez se encuentra localizada en un extenso valle a orillas del río Bravo, en el estado de Chihuahua, al otro lado de la ciudad de El Paso, Texas. Es uno de los centros maquiladores más importantes de México y junto con El Paso constituyen la región de mayor intercambio comercial del bloque económico formado por México, Estados Unidos y Canadá. Al igual que otras ciudades como Tijuana en Baja California, es un destino importante para los migrantes mexicanos que son deportados de Estados Unidos.

La metodología empleada se fundamenta en entrevistas semiestructuradas realizadas entre julio y octubre de 2019 a 23 hombres mexicanos deportados formalmente de EUA a Ciudad Juárez quienes manifestaron haber sido acusados de DUI. Todos los entrevistados son mayores de edad y fueron debidamente informados acerca de que su participación en este estudio era totalmente libre, de igual manera, para resguardar su identidad se les indicó que se usarían nombres ficticios en las publicaciones derivadas de esta investigación. Estas entrevistas buscan reconstruir las historias de vida de los migrantes deportados y se llevaron a cabo en cuatro lugares de Ciudad Juárez.

Se realizaron 12 de las 23 entrevistas en la Casa del Migrante que desde hace 39 años ofrece albergue a los migrantes que llegan a esa ciudad. En la Central de Autobuses se entrevistó a cinco deportados y en el Aeropuerto Internacional Abraham González se realizaron tres entrevistas más. En estos lugares, la identificación de los migrantes deportados partió de la observación de la vestimenta, la bolsa café de papel o transparente que dejaba ver los folders color manila que contenían los documentos de deportación expedidos por el Department of Homeland Security (Departamento de Seguridad Nacional, DHS, por sus siglas en inglés) y las constancias de repatriación del Instituto Nacional de Migración. Finalmente, en el Puente Santa Fe o Paso del Norte, que conecta Ciudad Juárez con la ciudad de El Paso, se realizaron las tres historias de vida restantes. Los migrantes deportados fueron detectados al bajar del puente y salir del módulo de repatriación del Instituto Nacional de Migración.

Las entrevistas semiestructuradas biográficas se construyen a partir del encadenamiento de hechos significativos denominados epifanías (Denzin \& Lincoln, 1995), turning points (Smith et al., 1998) o momentos críticos que han marcado la vida del entrevistado. A partir de esta metodología se reconstruye la vida de los entrevistados y se pone especial atención al proceso de deportación, su experiencia laboral y migratoria, antecedentes de delitos contra la ley de inmigración y abuso del alcohol u otras drogas. Un migrante deportado a Tijuana y que vivió casi toda su vida en Nueva York, ya que su madre lo llevó a vivir a esa ciudad cuando era niño, ofrece una perspectiva metodológica muy útil para valorar las ventajas y limitaciones de las historias de vida, cuando expresó lo siguiente: "algunas cosas no las voy a contar porque no me acuerdo, otras porque no quiero, y otras más porque todavía no las puedo superar, de ahí en adelante pregúntame lo que quieras" (Cárdenas Montaño \& Alarcón Acosta, 2017).

El artículo contiene tres apartados además de la introducción y conclusiones. En la introducción se presenta el planteamiento del problema de investigación, su objetivo central y la metodología. En el primer apartado se analizan los planteamientos teóricos más relevantes para guiar el análisis cualitativo. En el segundo apartado se describe el desarrollo histórico de la política de deportación masiva de personas no ciudadanas de Estados Unidos iniciada a mediados de la década de 1990. En el apartado tres 
se analizan los hallazgos principales de la investigación cualitativa para lo cual se describe el perfil sociodemográfico y migratorio de los 23 entrevistados, su proceso de deportación, la autoridad que llevó a cabo la detención, su experiencia laboral y migratoria, sus antecedentes penales y su experiencia en el abuso del alcohol $\mathrm{u}$ otras drogas. En la parte final del artículo se presentan las conclusiones de la investigación y sus implicaciones teóricas.

\section{Perspectivas teóricas sobre la deportación por DUI}

En esta sección se examinan las perspectivas teóricas y los hallazgos empíricos más significativos que se han hecho en investigaciones anteriores para analizar la información cualitativa sobre la deportación formal de ciudadanos mexicanos desde EUA bajo la acusación de haber cometido el delito de conducir un vehículo bajo la influencia del alcohol. Para este fin, analizamos primero la prevalencia del abuso del alcohol en eUA y México. Posteriormente, examinamos la investigación realizada en EUA sobre los factores que condicionan el abuso del alcohol entre los migrantes. Por último, acudimos a la teoría de la integración económica y social de los inmigrantes para analizar las trayectorias de integración de los migrantes mexicanos que llegan a ese país como menores de edad o como adultos.

De acuerdo con la Organización Mundial de la Salud (oms) (World Health Organization, 2018), la prevalencia de episodios de beber alcohol en abundancia ( heavy episodic drinking) afectó a una cuarta parte $(26.1 \%)$ de la población estadounidense general mayor de 15 años en comparación con $18.2 \%$ de la población mexicana similar en 2016. Esta prevalencia se define como consumir al menos 60 gramos de alcohol puro por lo menos en una ocasión en los últimos 30 días. En ambos países hay una diferencia significativa en esta prevalencia entre hombres y mujeres. En EUA, 45.1\% de los hombres tuvieron este tipo de abuso del alcohol y las mujeres tan solo $11.1 \%$. Para el caso de México, los porcentajes respectivos fueron 30.6\% y $6.1 \%$ en 2016.

La Encuesta Nacional de Consumo de Drogas, Alcohol y Tabaco (2016-2017) realizada en México define el abuso de alcohol como un patrón desadaptativo de consumo de esta sustancia que provoca un deterioro clínicamente significativo que conduce al incumplimiento de obligaciones, a problemas legales repetidos, a consumir alcohol de manera continuada y en situaciones peligrosas a pesar de tener problemas sociales o interpersonales continuos. Se considera que hay abuso de alcohol si uno o más de estos elementos se han presentado en un periodo de 12 meses. Los resultados de esta encuesta revelan que el patrón de consumo excesivo de alcohol en el último mes se incrementó de $12.3 \%$ a $19.8 \%$ entre 2011 y 2016. En el caso de los hombres, este consumo aumentó de $20.9 \%$ a $29.9 \%$ y en el de las mujeres de $4.1 \%$ a $10.3 \%$ (Villatoro et al., 2017).

La oms (World Health Organization, 2018) encontró diferencias importantes en relación con el tipo de bebidas consumidas, midiéndolas por litros de alcohol puro en 2016 o en el último año en el que hay información disponible. En EuA el alcohol fue consumido de la siguiente manera: cerveza $(47 \%)$, spirits $(35 \%)$ y vino $(18 \%)$. En México se encontró que la cerveza es, sin duda, la bebida alcohólica preferida por la población con $77 \%$, seguida muy de lejos por los spirits (20\%), el vino (2\%) y otras bebidas $(1 \%)$. Este hallazgo ya había sido documentado con anterioridad por 
Medina-Mora y colaboradores (2002), ya que en su investigación sobre México se encontró que la cerveza representa $63 \%$ del consumo de alcohol per cápita, seguida por los destilados (34\%) y en menor proporción los vinos de mesa (3\%). Estos autores argumentan que el consumo de alcohol no se distribuye de forma homogénea en la población ya que es consumido básicamente por hombres de edad media y $25 \%$ de los que más beben consumen $78 \%$ del alcohol disponible.

En relación con la experiencia de los migrantes mexicanos en EUA, el riesgo de que consuman alcohol y drogas en dicho país se asocia con su experiencia migratoria y puede estar relacionado con ciertos tipos de trabajos, con la duración de su permanencia fuera de México, con las experiencias de discriminación y el estrés asociado a su vida (Borges et al., 2007). Por otra parte, se argumenta que los inmigrantes mexicanos en EUA tienen un mayor riesgo de consumir drogas y de presentar trastornos por su uso debido a la amplia disponibilidad de drogas, además de la exposición a normas más liberales de uso de estas sustancias (Borges et al., 2013). Para Szaflarski y colaboradores (2011), los estados emocionales como la soledad, la nostalgia por tener esposa y familia en México, el sentido de compromiso por el trabajo y "perder los estribos" los fines de semana, están correlacionados con el abuso de sustancias y el estrés causado por el aislamiento social y las preocupaciones económicas.

Daniel-Ulloa y colaboradores (2014) identifican la relación de la embriaguez típica del fin de semana y el consumo excesivo de alcohol en los últimos 30 días en una muestra de trabajadores agrícolas latinos inmigrantes en Carolina del Norte. En este estudio se encontró que el nacimiento en México, el ingreso a EuA como adulto y la seguridad de un empleo durante todo el año están correlacionados con mayores probabilidades de la embriaguez de los fines de semana. En contraparte, una mayor aculturación y afiliación a una religión con prohibiciones estrictas contra el alcohol se asociaron con menores probabilidades de recurrir a una típica borrachera semanal.

Desde la perspectiva de la teoría de la integración económica y social de los inmigrantes en Estados Unidos, generalmente se asume que la asimilación es un proceso lineal, mediante el cual los inmigrantes se vuelven más similares al grupo dominante con el paso del tiempo, sin embargo, irónicamente, a mayor tiempo de residencia y exposición a Estados Unidos, mayor es la propensión de los inmigrantes a tener comportamientos de riesgo como la iniciación sexual más temprana y el abuso de sustancias como el alcohol (Rumbaut, 1997). De manera similar, Borges y colaboradores (2013) argumentan que entre los inmigrantes en general y los inmigrantes mexicanos en particular, a mayor duración de la residencia en EUA, mayor es el riesgo de trastornos por el uso de sustancias.

En efecto, el proceso de adaptación de los inmigrantes a la vida en EUA no es ni simple ni inevitable ya que tanto la población inmigrante como la sociedad receptora son heterogéneas. Los inmigrantes, aun los de la misma nacionalidad, pueden estar divididos por clase social, tiempo de llegada y generación. Asimismo, los inmigrantes pueden confrontar distintas situaciones que llevan a diversos resultados en términos de asimilación dependiendo del tiempo de arribo y el contexto de recepción (Portes \& Rumbaut, 2001).

Las trayectorias de integración o asimilación en EUA pueden ser muy diversas de acuerdo con la edad de arribo a ese país, es muy diferente ingresar siendo un niño que siendo una persona adulta. Rumbaut (1997) se refiere a los que ingresan siendo menores de edad como la "generación 1.5" para distinguirlos de la segunda generación que está compuesta por personas nacidas en Estados Unidos de padre o 
madre inmigrante. Rumbaut afirma que los niños de la "generación 1.5" se socializan y comienzan su educación primaria fuera de EuA y luego completan su educación en ese país. La edad de llegada de un inmigrante es un factor importante en su integración a la sociedad estadounidense ya que, por ejemplo, aquellos que llegan antes de los seis años (la "generación 1.75") son considerablemente más propensos a hablar inglés sin acento extranjero.

En su estudio de la segunda generación en EUA, cuyos resultados en esta investigación extendemos a la "generación 1.5", Portes y Rumbaut (2001) encuentran muchas contingencias y variables que cuestionan la asimilación como un camino uniforme y directo y la aparente conexión entre asimilación y movilidad social ascendente por lo que proponen la teoría de la asimilación segmentada. En este escenario, los inmigrantes difieren en tres dimensiones fundamentales. En primer lugar, importan sus características individuales, tales como, edad, educación, ocupación, habilidades, riqueza y conocimiento del inglés. En segundo lugar, es fundamental para su integración el entorno social que los recibe, incluidas las políticas gubernamentales, las actitudes de la población nativa y la presencia y tamaño de la comunidad coétnica. Finalmente, para la asimilación es muy importante la estructura familiar de los inmigrantes que refleja las culturas y las estructuras sociales del país de origen. En la estructura familiar es de particular importancia la presencia de los padres biológicos. Las perspectivas teóricas presentadas en esta sección son útiles para evaluar hasta qué punto es posible la integración económica y social exitosa de los inmigrantes mexicanos que residen en EUA bajo el status de indocumentados y que, por tanto, están sujetos a la deportación.

\section{La construcción y desarrollo de la "maquinaria de la deportación"}

En la larga historia de la migración masiva de México a Estados Unidos que se ha desarrollado a lo largo de más de un siglo, de acuerdo con Doris Meissner y colaboradores (2013, p. 93), los delitos relacionados con la inmigración habían sido tratados históricamente como violaciones de la ley civil que, en el peor de los casos, podrían llevar a la deportación que se reservaba para quienes habían sido convictos de ofensas criminales serias o violentas. Sin embargo, a partir de mediados de la década de 1990, el Congreso de ese país decidió facilitar la deportación de personas no ciudadanas al transformar las violaciones civiles de inmigración en crímenes y aumentar las penas para los crímenes de inmigración ya existentes. Por esta razón, entre 2003 y 2013, el número de procesamientos penales vinculados con violaciones relacionadas con inmigración crecieron a una tasa sin precedentes. Los dos crímenes de inmigración más procesados por los fiscales fueron el ingreso ilegal a Estados Unidos considerado antes como delito menor (misdemeanor) y el reingreso ilegal posterior a una remoción (illegal reentry) que es considerada ahora como un delito grave (felony). La categoría de no ciudadanos de Estados Unidos que están sujetos a la deportación formal incluye a personas indocumentadas, quienes tienen la residencia permanente legal o quienes son receptores de una visa temporal. Solamente los ciudadanos de Estados Unidos por nacimiento o naturalización no pueden ser deportados.

El DHs distingue dos tipos de deportaciones: remociones y retornos. En este artículo se usan de forma intercambiable los términos remociones o deportaciones 
formales. Las remociones (removals) son las expulsiones formales de extranjeros con base en una orden de remoción que tiene consecuencias administrativas o penales sobre reingresos subsecuentes a Estados Unidos. Entre las consecuencias legales que resultan de una remoción está la prohibición permanente para el reingreso a EUA para quienes han cometido delitos graves y la prohibición de cinco, 10 o 20 años para otro tipo de deportados. Los retornos (returns) son las expulsiones de extranjeros que no se fundamentan en una orden de remoción. La mayor parte de los retornos son de ciudadanos mexicanos que han sido aprehendidos en la frontera con México por la Patrulla Fronteriza (Border Patrol) y deportados a su país (U.S. Department of Homeland Security, 2011).

El endurecimiento del sistema judicial hacia los no ciudadanos se inició en 1988 con la Anti-Drug Abuse Act de ese año (Ley contra el Abuso de Drogas), la Anti-Terrorism and Effective Death Penalty Act de 1996 (Ley sobre Antiterrorismo y Pena de Muerte Efectiva) y la Illegal Immigration Reform and Immigrant Responsibility Act de 1996 (Ley de Reforma a la Inmigración Ilegal y la Responsabilidad del Inmigrante). Como resultado de estas leyes se ha expandido el procesamiento de crímenes relacionados con inmigración, así como el número de crímenes estatales y federales que conducen a la deportación formal o remoción. Asimismo, ha disminuido el criterio de los jueces de inmigración para suspender la remoción de no ciudadanos (Meissner et al., 2013, pp. 92-93). Todas estas transformaciones legislativas e institucionales llevaron a la creación de la "maquinaria de la deportación”, como la denomina Finnegan (2013).

Estas tres leyes someten a los no ciudadanos a la deportación obligada si cometen crímenes clasificados en una lista expandida de delitos agravados (aggravated felonies). También se aplican estándares más estrictos retroactivamente a crímenes por los que el castigo ya ha sido cumplido, como es el caso de la violencia doméstica y el Dui, y restringen seriamente la discreción judicial sobre la aplicación de la ley (Fitzgerald \& Alarcón, 2013, p. 120). Ahora existen cinco categorías diferentes de crímenes por los cuales los no ciudadanos, en caso de ser convictos, pueden ser deportados: crímenes de depravación moral, ofensas relacionadas con sustancias controladas, ofensas relacionadas con armas o dispositivos destructivos, crímenes de violencia doméstica o contra los niños y los delitos agravados (Meissner et al., 2013, p. 98).

Delitos agravados es un término que solo se usa en la ley de inmigración. En 1988, solamente había una lista de cuatro delitos de este tipo, sin embargo, con la promulgación de las tres leyes mencionadas, la definición del término se expandió significativamente hasta incluir alrededor de 50 crímenes entre los que se encuentran ofensas graves y relativamente menores, incluidos delitos clasificados anteriormente como no graves (misdeamenors) (Meissner et al., 2013, p. 98). En la lista de los delitos agravados se encuentran crímenes como asesinato, violación, abuso sexual de un menor, que atentan contra la vida y la integridad de las personas. También se incluye el tráfico de drogas, el tráfico ilícito de armas de fuego y el lavado de dinero. En los delitos contra la propiedad están el robo y el fraude. Hay algunos delitos agravados que tienen que ver directamente con violaciones a la ley de inmigración, tales como tráfico de extranjeros, reingreso de un extranjero previamente deportado (que en términos legales se denomina en inglés illegal reentry), falsificar o alterar un pasaporte y en esta misma línea también se incluye el fraude de documentos. Conducir bajo la influencia del alcohol u otras drogas (DUI) y conducir intoxicado (DWI) son considerados como crímenes de violencia, lo mismo que la agresión, el abuso infantil, la violencia doméstica, el homicidio involuntario, robo o resistencia al arresto. 
Aunque la "maquinaria de la deportación" ya estaba lista para operar con toda su fuerza desde el final de la década de 1990, fue hasta luego de ocurridos los atentados del 11 de septiembre de 2001 cuando se empezaron a incrementar las remociones de migrantes mexicanos que entran en el sistema judicial estadounidense. El 26 de noviembre de 2001, la Patriot Act se convirtió en ley y como consecuencia la inmigración a EUA se transformó en un asunto de seguridad nacional (Alarcón \& Becerra, 2012). En 2002, el gobierno estadounidense empezó a aplicar la sección 287 (g) de la Illegal Immigration Reform and Immigrant Responsibility Act (IIRIRA) de 1996 que permite al gobierno federal la posibilidad de establecer acuerdos con agencias policiales de ciudades y estados para entrenar a sus funcionarios como agentes de inmigración (U.S. Department of Homeland Security, 2011, p. 11). Las operaciones de detención de no ciudadanos en el interior de Estados Unidos que pueden conducir a la remoción, en 2003, quedó bajo la responsabilidad del Immigration and Customs Enforcement (ICE), mientras que la Customs Border Protection, que incluye a la Patrulla Fronteriza, realiza esta tarea en las fronteras.

El Programa de comunidades seguras fue iniciado por el presidente Bush y mantenido por su sucesor, el presidente Obama, hasta 2014. Este programa comenzó en octubre de 2008 y tiene por objeto crear una base de datos biométrica con capacidades de búsqueda de huellas digitales en todos los centros de arresto y detención que permite establecer el estatus migratorio de cada persona detenida al momento del arresto. Si la prueba biométrica muestra que la persona es deportable, los agentes del orden locales contactan al ICE. Esto permitía a las autoridades locales compartir con el gobierno federal las huellas digitales y otros datos biométricos de los indocumentados en cárceles locales y de los condados que se comparan con las bases de datos del FBI y el DHs. De acuerdo con el ICE, esto permite dar prioridad a la detención de los no ciudadanos que han cometido delitos graves. Las operaciones del Programa comunidades seguras fueron suspendidas temporalmente entre el 20 de noviembre de 2014 y el 25 de enero de 2017 cuando el presidente Trump ordenó su reactivación como resultado de la orden ejecutiva "Enhancing Public Safety in the Interior of the United States" ("Mejorando la seguridad pública en el interior de los Estados Unidos") (U.S. Immigration and Customs Enforcement, 2021).

Como consecuencia del fortalecimiento del aparato legislativo y judicial para facilitar las deportaciones formales de no ciudadanos, el número de los ciudadanos mexicanos deportados se incrementó exponencialmente. La Figura 1 contiene datos sobre deportación formal de ciudadanos mexicanos desde EuA, tomados de los Anuarios de Estadísticas de Inmigración del DHs entre 1998 y 2019, incluye los últimos tres años fiscales completos de la administración de Clinton (1998-2000), los ocho de la administración de Bush (2001-2008), los ocho años de la presidencia de Obama (20092016) y los primeros tres años completos de la administración de Trump (2017-2019). Estos datos revelan que el número de las deportaciones de ciudadanos mexicanos en este periodo llegó a $4616540 .^{1}$

\footnotetext{
${ }^{1}$ Las administraciones presidenciales en Estados Unidos inician el 20 de enero del primer año correspondiente, en la Figura 1 se incluyen los años completos de la administración por lo que no se agregan los últimos 20 días de enero cuando dejan el cargo.
} 
Figura 1. Número de ciudadanos mexicanos deportados formalmente desde Estados Unidos (1998-2019)

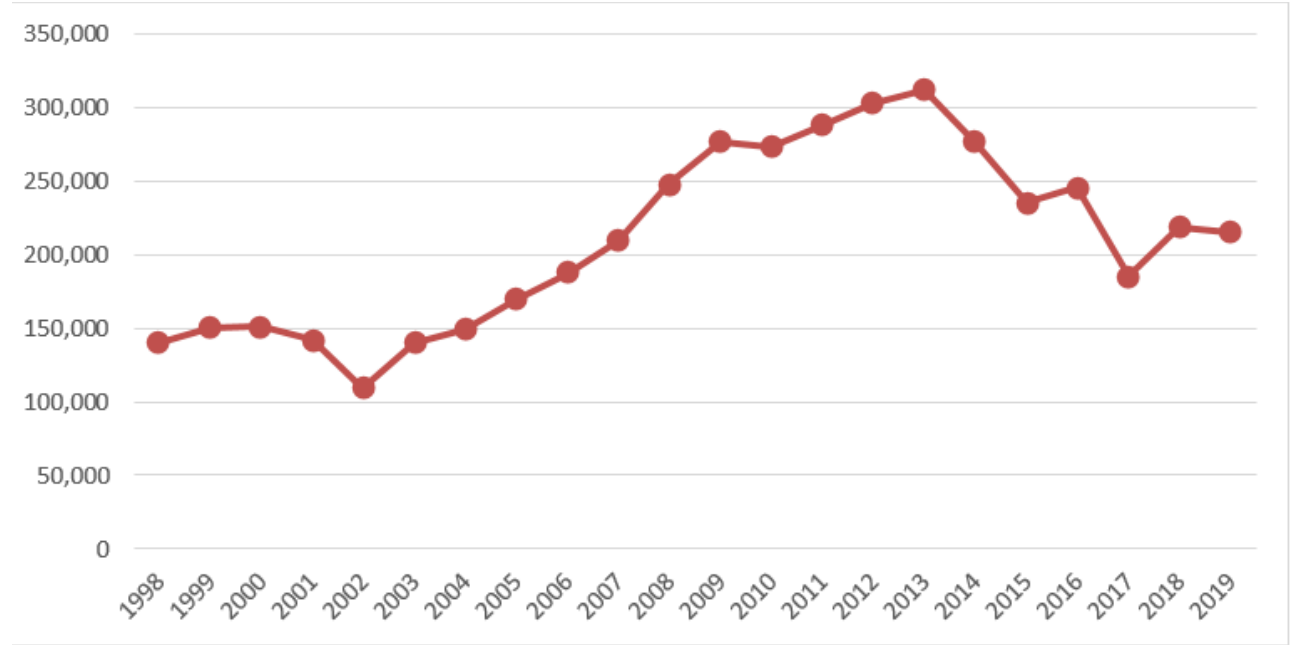

Fuente: U.S. Department of Homeland Security, 2020

Entre 1997 y 2000, durante la administración de Clinton, en el periodo posterior a la aprobación de IIRIRA, hubo alrededor de 150000 remociones de migrantes mexicanos por año. Justamente, después del 11 de septiembre de 2001, durante la presidencia de Bush, se inició un crecimiento espectacular en el número de deportaciones formales de migrantes mexicanos que llegó a 247000 en 2008, ya bajo la presidencia de Obama. Sin embargo, entre 2012 y 2013 se alcanzaron las cifras más altas de deportación, con 300000 mexicanos expulsados de Estados Unidos por año. A pesar de las amenazas de Trump, el número de deportaciones de los primeros tres años de su gestión fueron muy bajos en comparación con todo el periodo de Obama. La "maquinaria de la deportación" estaba preparada y bien aceitada desde el periodo de Obama, por lo menos en sus primeros tres años, el presidente Trump no la utilizó al máximo.

En enero de 2017, luego de iniciar su administración presidencial, Donald Trump empezó a emitir una gran cantidad de órdenes ejecutivas que afectaron las vidas de solicitantes de asilo y refugio, las personas nacidas en ciertos países musulmanes, los migrantes protegidos bajo el estatus de protección temporal y los jóvenes beneficiados por la Deferred Action for Childhood Arrivals (DACA), entre otros. En esta vorágine de medidas antiinmigrantes, el 25 de enero de 2017 se publicó la orden ejecutiva titulada "Enhancing Public Safety in the Interior of the United States" (The White House, 2017) en la que se expandieron las prioridades por las que cualquier no ciudadano podía ser removido del interior de ese país.

Entre los no ciudadanos que podían ser deportados formalmente se incluía no solo a los que habían sido convictos de un delito criminal, sino también a los que solamente habían sido acusados, quienes habían cometido un fraude en relación con su identidad en asuntos oficiales, quienes habían abusado de cualquier programa de recepción de beneficios públicos, quienes no habían salido de ese país después de recibir una orden final de remoción y quienes "a juicio de un oficial de inmigración constituían un riesgo para la seguridad pública o la seguridad nacional" (The White 
House, 2017). Con esta orden ejecutiva de Trump, casi cualquier persona que no es ciudadana de Estados Unidos podía ser deportada de ese país. Por otra parte, en 2019, se extendió la remoción expedita en el interior de EuA y se reactivó el Programa de comunidades seguras que había sido suspendido en la administración de Obama.

Como resultado de la pandemia ocasionada por SARS-CoV-2, el gobierno de EUA inició una serie de acciones relacionadas con la inmigración. En primer lugar, redujo una gran cantidad de operaciones administrativas que llevó a la reducción importante del número de remociones. Asimismo, ordenó la prohibición temporal de viajes "no esenciales" a través de sus fronteras. Este fue un acuerdo firmado con México el 20 de marzo de 2020. Ese mismo día, la administración de Trump anunció que se bloquearía el ingreso a los migrantes no autorizados y a los solicitantes de asilo debido a consideraciones de salud pública, sin importar su nacionalidad, quienes serían expulsados a México, Canadá, o a su país de origen. Estas expulsiones rápidas se han llevado a cabo bajo el título 42 del U.S. Code. Esta misma política ha sido continuada por la administración del presidente Biden, quien inició su gobierno en enero de 2021.

\section{Resultados}

La población de ciudadanos mexicanos deportados desde euA y que ahora residen en México es muy variada. Incluye más hombres que mujeres, personas que estuvieron en prisión, veteranos de las fuerzas armadas, jóvenes que fueron llevados a EuA por sus padres, migrantes que viven en situación de calle, los acusados de cometer delitos graves, los deportados por delitos leves como infracciones de tránsito o en redadas en lugares de trabajo, los deportados a partir de denuncias de sus empleadores o vecinos o después del reingreso ilegal.

En esta sección analizamos los casos de 23 migrantes mexicanos que fueron deportados de Estados Unidos a México a través de Ciudad Juárez, Chihuahua, acusados de conducir un vehículo bajo la influencia del alcohol u otras drogas y que fueron entrevistados entre julio y octubre de 2019 en los días siguientes a su expulsión del país vecino. Es importante reiterar que los entrevistados no provienen de una muestra aleatoria de migrantes deportados sino que aceptaron participar en la investigación luego de confirmar que habían sido deportados por Dur. En esta última sección se presentan los hallazgos empíricos más importantes de la investigación.

\section{El perfil sociodemográfico y migratorio de los 23 entrevistados en Ciudad Juárez}

Las entrevistas con los migrantes deportados ofrecen información sobre sus características sociodemográficas y migratorias más importantes, tales como, nombre ficticio, edad de último ingreso y deportación, número de años de residencia en Estados Unidos, estado civil, número de hijos y si algunos viven en la Unión Americana, así como estado de nacimiento en México. El promedio de edad de los entrevistados es de 35 años. De ellos $65 \%$ son padres de familia y sus hijos viven en EuA. Casi la mitad (48\%) está casado por lo que como consecuencia de la deportación hubo, en 
muchos casos, una ruptura del núcleo familiar. Los migrantes entrevistados nacieron principalmente en Michoacán, Oaxaca y Zacatecas (ver Tabla 1).

Tabla 1. Estado de nacimiento, edades de último ingreso y deportación y años de permanencia en Estados Unidos de migrantes entrevistados. Ciudad Juárez, 2019

\begin{tabular}{|c|c|c|c|c|c|c|c|c|}
\hline & $\begin{array}{l}\text { Nombre } \\
\text { ficticio }\end{array}$ & $\begin{array}{l}\text { Edad de } \\
\text { último } \\
\text { ingreso a } \\
\text { EUA }\end{array}$ & $\begin{array}{l}\text { Edad de de- } \\
\text { portación }\end{array}$ & $\begin{array}{l}\text { Años de per- } \\
\text { manencia en } \\
\text { EUA }\end{array}$ & $\begin{array}{l}\text { Estado de } \\
\text { nacimiento }\end{array}$ & Estado civil & $\begin{array}{l}\text { Con } \\
\text { hijos en } \\
\text { EUA }\end{array}$ & $\begin{array}{l}\text { Número } \\
\text { de hijos }\end{array}$ \\
\hline 1 & Manuel & 13 & 29 & 16 & Guanajuato & Soltero & No & 0 \\
\hline 2 & Jorge & 22 & 37 & 15 & Zacatecas & Separado & No & 0 \\
\hline 3 & Gabriel & 15 & 18 & 3 & Oaxaca & Soltero & No & 0 \\
\hline 4 & Alfredo & 5 & 43 & 38 & CDMX & Unión libre & Sí & 7 \\
\hline 5 & Martín & 17 & 38 & 21 & Michoacán & Separado & Sí & 2 \\
\hline 6 & Miguel & 16 & 31 & 15 & Puebla & Soltero & No & 0 \\
\hline 7 & Gerardo & 28 & 41 & 13 & Jalisco & Casado & Sí & 2 \\
\hline 8 & Esteban & 20 & 27 & 7 & Michoacán & Separado & No & 0 \\
\hline 9 & Federico & 11 & 42 & 31 & Guerrero & Casado & Sí & 7 \\
\hline 10 & Javier & 21 & 22 & 0.5 & Puebla & Soltero & Sí & 1 \\
\hline 11 & Mauricio & 14 & 48 & 34 & Oaxaca & Casado & Sí & 3 \\
\hline 12 & Isaías & 21 & 35 & 14 & Michoacán & Casado & Sí & 4 \\
\hline 13 & José & 17 & 34 & 17 & Michoacán & Casado & Sí & 4 \\
\hline 14 & Raúl & 24 & 34 & 10 & Michoacán & Casado & Sí & 1 \\
\hline 15 & Felipe & 47 & 52 & 5 & Sinaloa & Casado & No & 0 \\
\hline 16 & Inocencio & 13 & 18 & 5 & Veracruz & Soltero & No & 0 \\
\hline 17 & Arturo & 23 & 39 & 16 & Chihuahua & Casado & Sí & 6 \\
\hline 18 & Noé & 14 & 31 & 17 & Chiapas & Casado & Sí & 2 \\
\hline 19 & Ramiro & 25 & 45 & 20 & Zacatecas & Unión libre & Sí & 1 \\
\hline 20 & Juan & 17 & 44 & 27 & Michoacán & Casado & $\mathrm{Si}$ & 1 \\
\hline 21 & Eduardo & 13 & 25 & 12 & Michoacán & Soltero & No & 0 \\
\hline 22 & Francisco & 6 & 27 & 21 & Oaxaca & Unión libre & Sí & 2 \\
\hline 23 & Aurelio & 14 & 48 & 19 & Oaxaca & Casado & Sí & 3 \\
\hline
\end{tabular}

Fuente: Datos de entrevistas de trabajo de campo en Ciudad Juárez, 2019

Las consecuencias del proceso de deportación son muy distintas para quienes eran menores de edad cuando fueron llevados por sus familiares a Estados Unidos, pues se enfrentan a la situación de ser expulsados a un país que no conocen. Otras consecuencias afrontan los que emigraron de manera independiente y que llegaron a ese país siendo mayores de edad. La mayor parte de los entrevistados (61\%) llegó siendo menor de edad a EuA. Como ya se señaló, de acuerdo con Rumbaut (1997), estos inmigrantes pertenecen a la "generación 1.5", para distinguirlos de las personas que pertenecen a la segunda generación y que son los que nacieron en Estados Unidos de padre o madre inmigrante. Una información muy importante es que ninguno de 
ellos manifestó haber solicitado la DACA. Los nueve entrevistados restantes (39\%) ingresaron a ese país cuando ya eran adultos.

La edad promedio del último ingreso a Estados Unidos es 18 años, la edad promedio de deportación es 35 años y el promedio de tiempo de permanencia en ese país fue muy largo, 16 años. De las entrevistas se obtuvo información adicional. En lo que respecta a la educación, ninguno, ni los llegados como menores de edad o como adultos, terminó preparatoria o high school. Si a todo esto se añade que $96 \%$ de ellos se encontraba en situación indocumentada y solamente $4 \%$ tuvo la residencia permanente legal se puede comprender la dificultad de conseguir mejores empleos. El análisis de la última ocupación de los migrantes en EuA muestra que la mayor parte de los entrevistados se encontraba trabajando en la construcción (43.4\%), seguidos por la agricultura (9\%) y los restaurantes $(9 \%)$. En el resto, entre otros, Manuel era estudiante de inglés, Javier trabajó como coyote a cargo de las casas de seguridad y Francisco era supervisor en una florería.

\section{La deportación formal de Estados Unidos: antecedentes y consecuencias}

La mayor parte de los entrevistados comunicaron que tenían problemas más o menos graves de abuso de alcohol u otras drogas antes de su última deportación y que conducían habitualmente un vehículo motorizado bajo la influencia del alcohol u otras drogas. Por otra parte, como se observa en la Tabla 2, casi la mitad de ellos $(48 \%)$ tuvo una detención y/o deportación previa por DUi antes de ser deportados a Ciudad Juárez en 2019. Esta tabla también revela que dos de cada tres $(66 \%)$ fueron deportados directamente de la prisión por estar cumpliendo penas por delitos que cometieron, en la mayor parte de los casos, relacionados con DUI.

La prohibición para regresar a EuA después de una remoción puede variar desde cinco años, 10 años, 20 años a la expulsión de por vida, de acuerdo con las circunstancias de cada caso. En la Tabla 2 se muestra que el castigo por cometer Dur es generalmente de 10 años y esta sanción es la que se otorgó a 16 de los 23 entrevistados (69\%).

Ante la evidencia de que varios de los entrevistados fueron arrestados y/o deportados previamente a la última deportación, se presentan las historias de vida de Alfredo, Francisco y Manuel para mostrar cómo el abuso de alcohol u otras drogas los llevó a cometer delitos graves además de DUI y por esta razón estuvieron en prisión antes de la deportación.

Alfredo ingresó a Estados Unidos a los cinco años con su familia y llegó a residir a Yakima, Washington. "Me creía un ciudadano americano más". Cuando ya era adulto un amigo lo invitó a trabajar. Debía ir cada semana de Washington a Chicago a llevar droga. Lo hizo por 10 años y cree que ya lo estaba buscando la policía. Además, les ofrecía drogas a los paisanos recién llegados y les llevaba mujeres. "Mire, la gente que emigra solita se enreda en buscar algún vicio, no hay nada peor que la soledad, yo lucraba con ello, no me siento tan bien ahora". Lo detuvieron cuando regresaba de Chicago, en un retén cerca de su casa. Lo acusaron de manejar bajo la influencia del alcohol y fue deportado por 10 años, después de pasar dos años en prisión. 
Tabla 2. Estancias en prisión, arrestos, deportaciones previas y castigo a migrantes mexicanos deportados de Estados Unidos a Ciudad Juárez, 2019

\begin{tabular}{|c|c|c|c|c|}
\hline & Nombre & $\begin{array}{l}\text { Número de meses } \\
\text { en prisión }\end{array}$ & $\begin{array}{c}\text { Arrestos y/o deportaciones } \\
\text { previas por DUI }\end{array}$ & $\begin{array}{l}\text { Número de años de cas- } \\
\text { tigo por deportación }\end{array}$ \\
\hline 1 & Manuel & 36 & & De por vida \\
\hline 2 & Jorge & 12 & Orden de arresto por DUI & 10 \\
\hline 3 & Gabriel & 0 & & 10 \\
\hline 4 & Alfredo & 24 & & 10 \\
\hline 5 & Martín & 6 & $\begin{array}{l}\text { Orden de arresto y deportación } \\
\text { previa por DuI }\end{array}$ & 10 \\
\hline 6 & Miguel & 0 & & 10 \\
\hline 7 & Gerardo & 24 & & 10 \\
\hline 8 & Esteban & 0 & Deportación previa por DUI & 20 \\
\hline 9 & Federico & 0 & & 10 \\
\hline 10 & Javier & 0 & Deportación previa por DUI & 10 \\
\hline 11 & Mauricio & 12 & & 10 \\
\hline 12 & Isaías & 0 & & De por vida \\
\hline 13 & José & 6 & Dos arrestos por DUI & De por vida \\
\hline 14 & Raúl & 12 & $\begin{array}{l}\text { Arresto y deportación previa } \\
\text { por DUI }\end{array}$ & 10 \\
\hline 15 & Felipe & 24 & $\begin{array}{l}\text { Arresto por DUI y deportación } \\
\text { previa }\end{array}$ & De por vida \\
\hline 16 & Inocencio & 12 & $\begin{array}{l}\text { Orden de deportación previa } \\
\text { por DUI }\end{array}$ & 10 \\
\hline 17 & Arturo & 0 & Deportación previa por DUI & 10 \\
\hline 18 & Noé & 0 & & 10 \\
\hline 19 & Ramiro & 0 & & 10 \\
\hline 20 & Juan & 24 & & 10 \\
\hline 21 & Eduardo & 24 & Dos arrestos por DUI & De por vida \\
\hline 22 & Francisco & 36 & $\begin{array}{l}\text { Arresto y orden de deportación } \\
\text { previa por DuI }\end{array}$ & De por vida \\
\hline 23 & Aurelio & 12 & & 10 \\
\hline
\end{tabular}

Fuente: Entrevistas de trabajo de campo, Ciudad Juárez, 2019

Francisco nació en Oaxaca y llegó a Estados Unidos a la edad de seis años, posteriormente trabajó en una florería en Las Vegas, Nevada. Su padre fue deportado acusado de DUi. Tomaba alcohol en exceso y luego comenzó a consumir metanfetaminas y cristal. Lo detuvieron cuando salía de la casa de un amigo, "yo ya tenía una orden de arresto por no cumplir con la probation, por asalto y pelea sin arma, además de eso estaba tomado y había consumido algo de marihuana". Estuvo 36 meses en prisión y su deportación fue por DUI y orden de deportación previa. No puede regresar a Estados Unidos, "estoy fichado de por vida". 
Manuel nació en Guanajuato y estuvo viviendo en Tacoma, Washington, desde los 13 años. Trabajó en la irrigación del campo y junto con sus hermanos consiguió la residencia permanente legal que luego perdió con la deportación. Se comenzó a juntar con gente que iba a su casa y en la cochera ponían música, tomaban cervezas y todo iba bien hasta que se les ocurrió probar el cristal. En una ocasión sus amigos y él vieron un carro estacionado y se les hizo fácil robarlo.

A las dos semanas llegaron los policías a buscarme a la casa. Me detuvieron por haber robado el carro y por haber manejado supuestamente alcoholizado y terminé en la prisión, donde estuve tres años. No puedo creer que me deportaron de por vida.

A manera de contraste se describirán las historias de Esteban, Federico y Mauricio que, aunque fueron deportados por DUI, no cometieron otros delitos graves.

Esteban nació en Michoacán y estuvo viviendo en Las Vegas. Su último ingreso a Estados Unidos fue cuando tenía 20 años. Trabajaba haciendo mudanzas y los fines de semana manejaba tomado. "Mis amigos me decían que podía hacer cualquier cosa porque era güerito, ojo claro". La última vez que lo detuvieron iba manejando solo, al salir de una fiesta. "Ya no tenía gasolina, apenas llegué a la gasolinera y la señora que me atendió me comenzó a ver raro, hasta me dio el consejo de que no podía tomar y manejar". "Le dije sonriendo que no le estaba comprando cerveza sino gasolina y que ella no era la policía”. A las dos cuadras lo detuvo la policía. Duró tres días detenido y cuando lo iban a soltar, el ICE fue por él, porque tenía una deportación anterior por DUI, "me sacaron y me castigaron por 20 años".

Federico nació en Guerrero, cruzó a Estados Unidos en 1988, a los 11 años, y estuvo viviendo en Las Vegas. Los fines de semana tomaba cervezas con carne asada. Le tocaron varias redadas "encontraron que estaba medio tomado, me dejaban ir porque el alcoholímetro decía que no había sido tanto”. Una tarde al salir del trabajo se metió al freeway para irse a su casa.

Sentí que me venían siguiendo, yo creo que era porque mi camioneta estaba pintada de negro (...) muy elegante. Ganaba bastante bien en el área de cableado en la construcción. Al detenerme me revisaron y tuve que pagar una fianza de 1700 dólares por venir ligeramente tomado (...) no pudieron probar consumo de droga así que me mantuvieron detenido unos días por no tener papeles y porque mi caso era sospechoso.

En su hoja de deportación se puede leer que no puede regresar a Estados Unidos en 10 años.

Mauricio nació en la ciudad de Oaxaca, llegó a los 14 años a Estados Unidos y estuvo viviendo en Miami. El trabajo que hacía era ayudar en la reparación de barcos para lo cual era muy cuidadoso. "Como me vine chamaco, en México no tomaba, lo vine a hacer acá pero solo los fines de semana”. Fue detenido luego que tomó unas 15 cervezas y unas cuatro copas de tequila. Iba manejando solo ya que antes de salir de la reunión familiar dejó que su esposa saliera en otro carro con sus hijos. "Salí tomado y me detuvo la policía porque me quedé dormido esperando a que cambiara la luz del semáforo. Me llevaron directamente a detención, después de casi cuatro días me enviaron a Orlando, donde estuve 12 meses preso". En Estados Unidos tiene a su familia y asegura que va a regresar por ellos, lo deportaron castigado por 10 años. 


\section{Autoridad que llevó a cabo la detención previa a la deportación}

En las entrevistas con los 23 migrantes deportados surgió una información muy importante y es que la mayor parte de ellos $(69.5 \%)$ fue detenida por las policías locales, $21.7 \%$ por la Highway Patrol y $8.8 \%$ por el ICE. Esta información es corroborada por la Emif Norte que revela que, durante el trimestre de julio a septiembre de 2019, la mayor proporción de detenciones de mexicanos residentes en Estados Unidos fue realizada por policías locales (48.5\%), el ICE ocupa el segundo lugar con $24.2 \%$ de las detenciones. El resto de detenciones $(27.3 \%)$ fue llevado a cabo por otras agencias de aplicación de la ley (El Colegio de la Frontera Norte, 2019). Sorprende mucho que ICE, la agencia del DHS, que tiene a su cargo la deportación de no ciudadanos en el interior de ese país haya detenido el porcentaje más bajo de los entrevistados. Por su parte, Rocha Romero y Ocegueda Hernández (2013, pp. 16-31), en su investigación sobre mujeres deportadas a Tijuana y que fueron entrevistadas en el albergue Madre Assunta, señalan que las detenciones realizadas a diez mujeres entrevistadas no se hicieron por agentes del ICE sino por policías locales. Estas detenciones se llevaron a cabo en lugares de reunión de la comunidad inmigrante mexicana en Estados Unidos, como tiendas, lugares de esparcimiento o centros religiosos.

El alto número de detenciones y deportaciones de ciudadanos mexicanos en el interior de Estados Unidos muestra la colaboración activa de autoridades subnacionales en tareas de control migratorio a través de los programas 287 (g) y comunidades seguras. Como ya se indicó en párrafos anteriores, el programa 287(g) es una sección de la Ley de Inmigración y Nacionalidad que desde 1996 permite al gobierno federal la posibilidad de establecer acuerdos con agencias policiales de ciudades y estados para entrenar a sus funcionarios como agentes de inmigración (Meissner et al., 2013). El Programa de comunidades seguras, iniciado por el presidente Bush en 2008, fue mantenido en operación por el presidente Obama hasta 2014 y luego reactivado por el presidente Trump en 2017, tiene como finalidad compartir con el gobierno federal las huellas digitales y otros datos biométricos de los indocumentados en cárceles locales y de los condados que se comparan con las bases de datos del FBI y el DHS (U.S. Immigration and Customs Enforcement, 2021).

Las experiencias de detención de Raúl, Noé y Ramiro, que se presentan en las siguientes líneas, muestran la forma cómo estos tres migrantes mexicanos fueron detenidos previamente a la deportación por agentes de las policías locales. De hecho, en los seis casos mostrados son también agentes de policías locales los que realizan la detención, muchas veces en retenes en la vía pública, lo que pone en evidencia la efectividad de los programas 287 (g) y comunidades seguras. Por otro lado, la mayoría de los entrevistados fueron detenidos cuando estaban alcoholizados y generalmente por haber bebido cerveza.

Raúl nació en Michoacán e ingresó a Estados Unidos a la edad de 24 años. Estuvo viviendo en Arizona por 10 años, trabajaba en la construcción. Cuando salía del trabajo la policía lo detuvo por haber tomado

un poquito, lo hice y pagué la fianza, sentí que me iban a dejar salir como la vez pasada pero el ICE fue por mí para deportarme por ser indocumentado, que porque tenía un caso abierto. Los cargos que me echaron fueron por alcohol y droga (...) nunca figuró mi falta de papeles y yo no le hago a la droga. Estuve casi un año en la cárcel y no puedo regresar en 10 años. 
Noé nació en Chiapas, su último ingreso a Estados Unidos fue cuando tenía 14 años. Estuvo viviendo en Carolina del Norte donde trabajaba en un campo de golf. Cuando lo detuvieron iba manejando hacia su trabajo. La policía lo iba siguiendo y le ordenó que se detuviera. El policía le preguntó si estaba tomando y él dijo que sí porque tenía cervezas abiertas dentro del carro. "Creo que mi copiloto estuvo tomando cerveza". Lo detuvieron por dos días, "mi familia estaba tratando de sacarme, pero me entregaron al ICE rápidamente, una vez ahí es más difícil que te saquen”. Lo llevaron a la prisión de Georgia y de ahí lo deportaron por 10 años.

Ramiro nació en Zacatecas e ingresó a Estados Unidos a la edad de 25 años. Cuando lo detuvieron en el Paso, Texas, fue "por algo tonto, al subirme al carro no prendí las luces, era de noche, pero no había nadie en el camino". Al pararlo, los policías olieron el alcohol, se había tomado dos cervezas.

Me pidieron mi licencia y no traía ni un papel. Le dije al oficial que había tomado un par de cervezas antes de que empezara el partido de futbol, ni modo que no me creyera, traía mi uniforme, él me dijo que si hubiera traído licencia me hubiera dejado ir.

Por la deportación, no puede regresar a Estados Unidos hasta que pasen 10 años.

\section{Conclusiones}

Esta investigación tuvo como objetivo central analizar los factores legislativos, sociales y económicos que explican la razón por la que un alto número de ciudadanos mexicanos han sido deportados formalmente desde Estados Unidos bajo la acusación de conducir un vehículo bajo la influencia del alcohol. Para cumplir con este objetivo se examinaron las teorías y las investigaciones más pertinentes que pudieran ayudar a entender este proceso, así como la política de inmigración de Estados Unidos que en la actualidad facilita la deportación formal de una gran cantidad de extranjeros y que ha afectado en buena medida a los ciudadanos mexicanos. El marco teórico y contextual fundamenta el análisis de la investigación empírica que consistió en examinar los procesos de deportación de 23 hombres mexicanos que fueron removidos de Estados Unidos a Ciudad Juárez, Chihuahua, acusados de Dur en 2019.

El primer hallazgo fundamental de la investigación es que de acuerdo con la oms (World Health Organization, 2018) el abuso en el consumo del alcohol fue más alto en la población estadounidense mayor de 15 años en comparación con la población mexicana en 2016. Esta información sugiere que los migrantes mexicanos al ingresar a Estados Unidos se ven expuestos a una práctica más extendida en el abuso de alcohol que la que hay en México. Es probable que, por esta razón, la mayor parte de los entrevistados iniciaron el consumo de alcohol en ese país, aunque hay que señalar que la mayoría de ellos fueron llevados cuando eran menores de edad por sus padres. Otro hallazgo muy importante es que, tanto en México como en Estados Unidos, la cerveza es la bebida alcohólica preferida. Casi todos los entrevistados informaron que la consumían habitualmente y que incluso abusaban de su consumo y que esto los llevó a la deportación. La popularidad de la cerveza en México ha sido documentada en investigaciones realizadas por la oms (World Health Organization, 2018) y por Medina-Mora y colaboradores (2002). 
Dentro del perfil sociodemográfico y migratorio se encontró que los entrevistados no pueden considerarse jóvenes ya que el promedio general de edad es de 35 años. Como era de esperar, ninguno de ellos es ciudadano de Estados Unidos, la mayoría tenía estatus indocumentado y solamente $4 \%$ tenía residencia permanente legal, que fue cancelada al momento de su deportación. Por otra parte, la totalidad de los entrevistados tienen escolaridad muy baja ya que ninguno, ni los llegados como menores de edad, terminó la preparatoria o high school. Es quizás por esta razón que ninguno de ellos solicitó la protección de DACA contra la deportación por no cumplir con el requisito de educación que requería esta orden ejecutiva. La mayoría informó haber tenido empleos relacionados con la construcción, la agricultura y los restaurantes.

Los entrevistados decidieron conducir un automóvil para ir a trabajar y para realizar las otras actividades en su vida cotidiana a pesar de haber consumido distintas cantidades de alcohol y fueron detenidos, en la mayoría de los casos, por oficiales de policías locales. Este es otro hallazgo relevante y documenta el papel protagónico de estos oficiales de departamentos de policía locales que actúan como "policías de inmigración". Esta acción es resultado directo de la aplicación de los programas $287(\mathrm{~g})$ y comunidades seguras que empezaron a operar luego de la aprobación por el Congreso de la Illegal Immigration Reform and Immigrant Responsibility Act en 1996 que transformó al DuI en un delito agravado que puede conducir directa y retroactivamente a la deportación formal (Meissner et al., 2013).

Otro resultado de esta investigación con implicaciones teóricas se relaciona con el largo promedio de años vividos en Estados Unidos por los entrevistados: 16 años. Como consecuencia de esto, muchos de los inmigrantes deportados tienen hijos en ese país, están casados o en unión libre. La larga residencia en EuA lleva a relacionar los hallazgos con la teoría sobre el proceso de integración económica y social de los inmigrantes que muchos académicos estadounidenses denominan asimilación. En general se asume que la asimilación es un proceso uniforme y directo en el que participan todos los inmigrantes de Estados Unidos y que inexorablemente conduce a la movilidad social ascendente con el paso del tiempo. Portes y Rumbaut (2001) cuestionan este postulado y, siguiendo la teoría de la asimilación segmentada, argumentan que algunos inmigrantes en lugar de alcanzar la movilidad social ascendente viven en condiciones de pobreza en el segmento inferior y degradado de la sociedad estadounidense. En este contexto, Rumbaut (1997) destaca la ironía de que a mayor tiempo de residencia y exposición a la vida en EUA, mayor es la propensión de los inmigrantes a tener comportamientos de riesgo como el abuso del alcohol.

Este tipo de integración económica y social degradada es la que experimentaron los entrevistados, la mayor parte de los cuales vivieron en ese país de forma indocumentada. Esto los llevó en la mayoría de los casos a vivir en comunidades de hombres solos que se emplean en un mercado de trabajo precario y bajo un ambiente social de discriminación. Investigaciones sobre el alcoholismo señalan que esta situación conduce al abuso del alcohol. Para Szaflarski y colaboradores (2011), la soledad, la nostalgia, el estrés causado por el aislamiento social y las preocupaciones económicas están correlacionadas con el abuso de sustancias. Borges y colaboradores (2007) argumentan que los inmigrantes mexicanos tienen un mayor riesgo de consumir drogas porque en Estados Unidos hay mayor disponibilidad de estas substancias y normas más liberales en su uso.

Los 23 entrevistados tenían distintos niveles en el abuso de alcohol o drogas desde antes de la deportación que causó que casi la mitad de ellos tuviera una detención 
y/o deportación previa por Dui antes de ser deportados a Ciudad Juárez en 2019. La práctica de abuso de alcohol u otras drogas en espacios y tiempos de trabajo y convivencia con familiares y amigos se convirtió en delito cuando condujeron un vehículo motorizado bajo la influencia de esta substancia que puso sus vidas y las de otras personas en peligro. Este delito grave lleva a castigos severos a ciudadanos de Estados Unidos que pueden perder el privilegio de tener una licencia de manejar y/o terminar en prisión, sin embargo, para los no ciudadanos este delito puede conducir directamente a la deportación. En este sentido, Hagan y colaboradores (2011, pp. 1388-1389) señalan que la deportación se ha convertido en una forma de control social ya que no solamente se exige que los inmigrantes sean residentes legales, sino que adopten pautas de conducta sin precedentes a riesgo de enfrentar la deportación permanente. Con esta investigación se encontró que el abuso del alcohol afecta a muchos migrantes mexicanos que residen en Estados Unidos, por esta razón, se hace necesario hacer un trabajo de prevención que cuente con la participación de las mismas comunidades de migrantes, las iglesias y los consulados mexicanos.

\section{Referencias}

Alarcón, R. \& Becerra, W. (2012). ¿Criminales o víctimas? La deportación de migrantes mexicanos de Estados Unidos a Tijuana, Baja California. Norteamérica, 7(1), 125-148. https://www.revistanorteamerica.unam.mx/index.php/nam/ article/view/160

Borges, G., Medina-Mora, M. E., Breslau, J. \& Aguilar-Gaxiola, S. (2007). The effect of migration to the United States on substance use disorders among returned Mexican migrants and families of migrants. American Journal of Public Health, 97(10), 1847-1851. https://doi.org/10.2105/AJPH.2006.097915

Borges, G., Rafful, C., Tancredi, D. J., Saito, N., Aguilar-Gaxiola, S., Medina-Mora, M. E. \& Breslau, J. (2013). Mexican immigration to the us, the occurrence of violence and the impact of mental disorders. Brazilian Journal of Psychiatry, 35(2), 161-168. https://doi.org/10.1590/1516-4446-2012-0988

Cárdenas Montaño, M. \& Alarcón Acosta, R. (2017). Historias de vida de deportados, centroamericanos en tránsito y solicitantes de asilo en Casa del Migrante de Tijuana (2013-2016). En Casa del Migrante en Tijuana, A. C., Vidas en vilo. Historias y testimonios de migrantes internacionales (pp. 101-215). Ediciones ILCSA. https://www.academia.edu/42738022/_Historias_de_vida_de_deportados_centroamericanos_en_tr\%C3\%A1nsito_y_solicitantes_de_asilo_en_Casa_ del_Migrante_de_Tijuana_2013_2016_

Coubès, M.-L. (2018). Deportaciones de mexicanos desde Estados Unidos: ¿qué está cambiando con el nuevo gobierno de Estados Unidos? Coyuntura Demográfica, (13), 87-95. http://coyunturademografica.somede.org/wp-content/plugins/ coyuntura_demografica/DEMOGRAFICA/ARTICULOS/PUB-2018-13-152.pdf

Daniel-Ulloa, J., Reboussin, B. A., Gilbert, P. A., Mann, L., Alonzo, J., Downs, M. \& Rhodes, S. D. (2014). Predictors of heavy episodic drinking and weekly drunkenness among immigrant Latinos in North Carolina. American Journal of Men's Health, 8(4), 339-348. https://doi.org/10.1177/1557988313519670 
Denzin, N. K. \& Lincoln, Y. S. (1995). Editors' Introduction. Qualitative Inquiry, 1(1), 3-6. https://doi.org/10.1177/107780049500100101

EFE. (2019, 22 de mayo). El DUI, la herramienta "exprés" del gobierno para deportar inmigrantes. https://www.efe.com/efe/usa/inmigracion/el-dui-la-herramienta-expres-del-gobierno-para-deportar-inmigrantes/50000098-3982603

El Colegio de la Frontera Norte. (2019, julio-septiembre). Boletín Emif Norte. Encuesta sobre Migración en la Frontera Norte de México. Reporte trimestral de resultados. https:/ / www.colef.mx/emif/datasets/boletines/norte/2019/Emif\%20Norte\%20Boletin\%20T3\%202019.pdf

Finnegan, W. (2013, 22 de abril). The deportation machine. A citizen trapped in the system. The New Yorker (Annals of immigration, 29 de abril). https:/ /www.newyorker.com/magazine/2013/04/29/the-deportation-machine

Fitzgerald, D., \& Alarcón, R. (2013). Migration: policies and politics. En P. H. Smith \& A. Selee (Eds.), Mexico and the United States: The politics of partnership (pp. 111138). Lynne Rienner Publishers.

Hagan, J. M., Rodriguez, N. \& Castro, B. (2011). Social effects of mass deportations by the United States government, 2000-10. Ethnic and Racial Studies, 34(8), 13741391. https://doi.org/10.1080/01419870.2011.575233

Medina-Mora, M. E., Natera, G. \& Borges, G. (2002). Alcoholismo y abuso de bebidas alcohólicas. Observatorio mexicano en tabaco, alcohol y otras drogas. Conadic-Secretaría de Salud México. http://conadic.salud.gob.mx/pdfs/publicaciones/ obs2002_1.pdf

Meissner, D., Kerwin, D. M., Chishti, M. \& Bergeron, C. (2013). Immigration enforcement in the United States: The rise of a formidable machinery. Migration Policy Institute. https://www.migrationpolicy.org/sites/default/files/publications/enforcementpillars.pdf

Portes, A. \& Rumbaut, R. G. (2001). Legacies: the story of the immigrant second generation. University of California Press.

Rocha Romero, D. \& Ocegueda Hernández, M. T. (2013). Después de tantos años me deportaron. Proceso de identificación y deportación de mujeres inmigrantes no delincuentes. Estudios Fronterizos, 14(28), 9-34. https://doi.org/10.21670/ ref.2013.28.a01

Rumbaut, R. G. (1997). Assimilation and its discontents: between rhetoric and reality. International Migration Review, 31(4), 923-960. https://doi.org/10.2307/2547419

Smith, P. (1998). The new American cultural sociology. Cambridge University Press. https://doi.org/10.1017/CBO9780511520808

Szaflarski, M., Cubbins, L. A., \& Ying, J. (2011). Epidemiology of alcohol abuse among us immigrant populations. Journal of Immigrant and Minority Health, 13(4), 647658. https://doi.org/10.1007/s10903-010-9394-9

The White House. (2017, 30 de enero). Enhancing public safety in the interior of the United States. Federal Register, 82(18), pp. 8799-8803. https://www.govinfo.gov/ content/pkg/FR-2017-01-30/pdf/2017-02102.pdf

TRAC Immigration. (2021). Latest data: Immigration and Customs Enforcement removals. Recuperado el 12 de septiembre de 2021 de https://trac.syr.edu/phptools/immigration/remove/ 
U.S. Department of Homeland Security. (2011). Yearbook of Immigration Statistics: 2011. https://www.dhs.gov/immigration-statistics/yearbook/2011

U.S. Department of Homeland Security. (2020, 16 de septiembre). Yearbook of Immigration Statistics: 2019. https://www.dhs.gov/immigration-statistics/yearbook/2019

U.S. Immigration and Customs Enforcement. (2021). Secure communities. https://www. ice.gov/secure-communities

Villatoro, J., Resendiz, E., Mujica, A., Bretón, M., Cañas, V., Soto, I., Fregoso, D., Fleiz, C., Medina, M., Gutiérrez, J., Franco, A., Romero, M. \& Mendoza, I. (2017). Encodat. Encuesta Nacional de Consumo de Drogas, Alcohol y Tabaco 2016-2017. Reporte de alchool (p. 190). Instituto Nacional de Psiquiatría Ramón de la Fuente/Instituto Nacional de Salud Pública/Comisión Nacional contra las Adicciones/Secretaría de Salud. https://drive.google.com/file/d/1rMlKaWy34GR51sEnBK2-u2q_ BDK9LA0e/view

World Health Organization. (2018, 27 de septiembre). Global status report on alcohol and health 2018. https://www.who.int/publications-detail-redirect/9789241565639

Karen Muro Aréchiga

Mexicana. Maestra en estudios de migración internacional por El Colegio de la Frontera Norte en Tijuana, B.C. Es investigadora independiente. Líneas de investigación: los procesos de deportación de mexicanos de Estados Unidos. Publicación reciente: Muro Aréchiga, K. (2019). Mexicanos deportados de Estados Unidos a Ciudad Juárez por conducir bajo la influencia del alcohol u otras drogas [Tesis de maestría, El Colegio de la Frontera Norte].

Rafael Alarcón

Mexicano. Doctor en planeación urbana y regional por la Universidad de California, Berkeley. Pertenece al Sistema Nacional de Investigadores en el nivel III. Profesor investigador del Departamento de Estudios Sociales en El Colegio de la Frontera Norte. Líneas de investigación: el impacto económico y social de la migración de México a Estados Unidos, la integración de los inmigrantes, la migración calificada y la deportación. Publicación reciente: Escala, L., Odgers, O. \& Alarcón, R. (2016). Making Los Angeles home. The integration of Mexican immigrants in the United States. University of California Press. 